\title{
CAMP-Dependent Protein Kinase Catalytic
} Subunit Gamma

National Cancer Institute

\section{Source}

National Cancer Institute. cAMP-Dependent Protein Kinase Catalytic Subunit Gamma.

NCI Thesaurus. Code C38414.

CAMP-dependent protein kinase catalytic subunit gamma (351 aa, $40 \mathrm{kDa}$ ) is encoded by the human PRKACG gene. This protein plays a role in both CAMP-mediated signaling and protein phosphorylation. 